This PDF is a selection from an out-of-print volume from the National Bureau of Economic Research

Volume Title: Annals of Economic and Social Measurement, Volume 3, number 1

Volume Author/Editor: Sanford V. Berg, editor

Volume Publisher: NBER

Volume URL: http://www.nber.org/books/aesm74-1

Publication Date: 1974

Chapter Title: A Method for Stochastic Control of Nonlinear Econometric Models and an Application: Abstract

Chapter Author: J. Phillip Cooper, Stanley Fischer

Chapter URL: http://www.nber.org/chapters/c10003

Chapter pages in book: (p. 205 - 206) 


\section{A METHOD FOR STOCHASTIC CONTROL OF NONLINEAR ECONOMETRIC MODELS AND AN APPLICATION: ABSTRACT}

\section{By J. Phillip Cooper and Stanley Fischer}

In this paper, we describe a method for controlling stochastic, nonlinear, econometric models and illustrate the method by studying monetary and fiscal policy in the St. Louis econometric model. The method consists essentially of estimating the means and variances of the dynamic responses of the nonlinear model to changes in instrument variables, in order to produce a linear representation of the model with random coefficients, and then using this linear, stochastic version in optimization. The major virtue of the method is that it is as easily applied to large as to small models.

In more detail, the method has five steps. First, choose nominal paths for the control variables in the problem. Second, carry out a series of stochastic simulations of the model being optimized; in these simulations, stochastic elements of the model are generated by a pseudorandom number generator and stochastic disturbances are generated around the nominal paths of the control variables. Third, calculate linear regressions in which the simulated target variables of the model are the dependent variables and the instrument variables are among the independent variables of the regressions. Fourth, compute optimal feedback control rules for the instrument variables using the linear regressions calculated at the third step to represent the model. Fifth, evaluate these feedback control rules in stochastic simulations of the original model.

The nominal paths chosen for the instruments in our work were simple constant growth rate paths; in principle, these nominal paths could also be obtained as the solution to a deterministic, nonlinear control problem. In the second step, the underlying model is treated as stochastic; the stochastic disturbances of the instrument variables around the nominal paths have the same variance as historical experience. As a result of the second step, we have a large set of data representing the response of the model to changes in policy. The third step summarizes this information in linear, random coefficients regressions, consisting of parsimonious, autoregressive-moving average models representing the target variables as functions of current and lagged instruments and lagged target variables. A quadratic loss function is used at the fourth step to obtain optimal feed back control rules for the linearized version of the model represented by the linear regressions. The fifth step is undertaken as a check on the operation of the rules in the original model.

In our work with the St. Louis model, we took the rates of inflation and unemployment to be target variables, and the growth rates of money and high employment federal expenditures to be instruments. The control rules produced were applied in twenty replications of a stochastic simulation of the original model in which both coefficients and additive errors were treated as random. The control rules produced losses significantly below those obtained under constant growth rate rules for the instruments and historical experience; changing the relative 
weights on arguments in the loss function produced the expected changes in the results of policy. For example, reducing the cost of deviations of unemployment from its desired level reduced the average deviation of inflation from its target level. Comparison of the optimal rule obtained in this paper with the best heuristic rule found in Cooper and Fischer (1973) showed that losses due to deviations of the rates of inflation and unemployment from their target values obtained here were similar to those in our previous paper but with less variation in the instruments. It is also shown that while fiscal policy contributes little added stabilization when an active monetary policy is used, fiscal policy does stabilize the model if the money supply grows at a constant rate.

University of Chicago

\section{REFERENCE}

Cooper, J. Phillip and Stanley Fischer, "Monetary and Fiscal Policy in the Fully Stochastic St. Louis Model," in Journal of Money, Credit and Banking, (February, 1974). 